\title{
Measurements of inclusive vector boson production from ATLAS
}

\author{
Mikhail Karnevskiy ${ }^{1, a}$, on behalf of the ATLAS collaboration \\ ${ }^{1}$ Johannes Gutenberg University of Mainz, Germany
}

\begin{abstract}
The measurements of single $W, Z$ boson production in association with jets probes QCD in a multi-scale environment. In this paper, we summarize the latest measurements of $W+$ jet and $Z+$ jet production and their ratio, extending to high jet multiplicities over a large set of kinematic distributions for proton-proton collisions at a center of mass energy of $7 \mathrm{TeV}$ data at the LHC. The results are compared to the latest theoretical predictions of modern Monte Carlo generators. In addition, the measurement of production of vector bosons in association with heavy flavours allows to study c and b-quark parton distribution functions (PDFs) and gluon splitting effects. Also the corresponding measurements of $W+c$ and $Z+b(b)$ are reported and compared to predictions based on various PDFs in four and five-flavour schemes.
\end{abstract}

\section{Introduction}

The production of $W$ and $Z$ bosons is well understood and provides a clear experimental signal in the leptonic decay channels into electrons $e$ and muons $\mu$, which allow for the most precise cross-section measurements. This short review presents several recent measurements of $Z$ boson, performed at the ATLAS detector. These are based on $7 \mathrm{TeV}$ and $8 \mathrm{TeV}$ data-set with an integrated luminosity of $L \sim 4.6 \mathrm{fb}^{-1}$ in 2011 and $\sim 20 \mathrm{fb}^{-1}$ in 2012 , respectively. A more detailed review of the single and di-boson production at the $\mathrm{LHC}$ can be found in [1].

The measurement of the Drell-Yan process of di-lepton production in bins of invariant mass is sensitive to the parton distribution functions (PDFs), in particular to the less known distribution of anti-quarks at large Bjorken- $x$ values. In addition, the differential cross section $d \sigma / d m$ is theoretically described by perturbative QCD (pQCD). Hence a comparison of precisely measured experimental data with theoretical predictions provides a crucial test of pQCD. The di-lepton mass spectrum was measured at the ATLAS experiment at $\sqrt{s}=7 \mathrm{TeV}$ in several mass regions. The corresponding measurements are shown in Sec. 2.

The leptons produced in the annihilation process $q \bar{q} \rightarrow Z / \gamma^{*} \rightarrow l^{+} l^{-}$, are asymmetric with respect to the quark direction in the rest frame of the di-lepton system. The forward-backward asymmetry $A_{F B}$, which is sensitive to the electroweak mixing able $\sin ^{2} \theta_{W}$, of this process is defined as

$$
A_{F B}=\frac{\sigma_{\cos \theta_{C S}^{*}>0}-\sigma_{\cos \theta_{C S}^{*}<0}}{\sigma \cos \theta_{C S}^{*}>0+\sigma_{\cos \theta_{C S}^{*}<0}}
$$

\footnotetext{
ae-mail: karnevsk@uni-mainz.de
} 


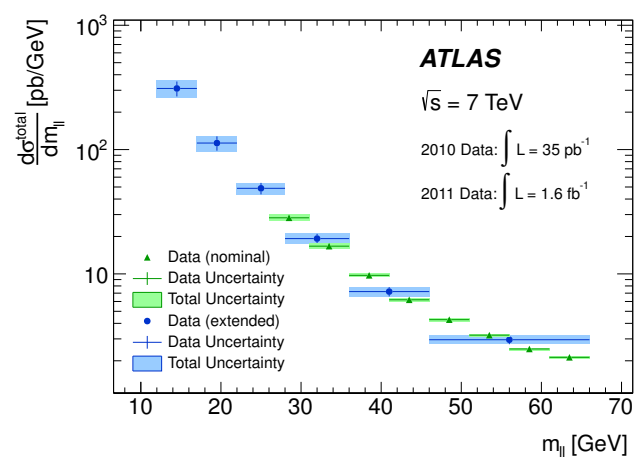

Figure 1. Comparison of Born-level nominal $(e+\mu)$ and extended $(\mu)$ channel differential cross sections as a function of the di-lepton invariant mass, $m_{l l}$, extrapolated to full phase space. The data uncertainties are the total fiducial cross-section uncertainties, while the total uncertainties also include theoretical uncertainties from the acceptance correction [2].

using angle in the Collins-Soper frame,

$$
\cos \theta_{C S}^{*}=\frac{p_{l l}^{z}}{\left|p_{l l}^{z}\right|} \frac{2\left(p_{1}^{+} p_{2}^{+}-p_{1}^{+} p_{2}^{+}\right)}{m_{l l} \sqrt{m_{l l}^{2}+p_{T, l l}^{2}}}
$$

with $p_{i}^{ \pm}=\frac{E_{i} \pm p_{z, i}}{\sqrt{2}}$. The measurement of $A_{F B}$ at the ATLAS detector [4] is summarized in Sec. 3 .

The transverse momentum $\left(p_{T}\right)$ distribution of $W$ and $Z$ bosons produced in hadronic collisions is a further probe of strong interaction dynamics. The low transverse momentum range is governed by initial-state parton radiation (ISR), but also due to the intrinsic transverse momentum of the initialstate partons inside the proton, while quark-gluon scattering dominates at high $p_{T}$ regime and is described by pQCD. A good modeling of the vector boson $p_{T}$ distribution in Monte Carlo generators is highly important in many physics analyses at the LHC, such as the precision measurement of the $\mathrm{W}$ boson mass. The corresponding measurements are summarized in 4 .

\section{Di-lepton invariant mass}

The measurement of di-lepton in the low mass region was performed in the $e$ and $\mu$ channels for invariant masses between $26 \mathrm{GeV}$ and $66 \mathrm{GeV}$ using an integrated luminosity of $1.6 \mathrm{fb}^{-1}$ collected in 2011. The measurements of both decay channels have been combined. The analysis is extended to invariant masses as low as $12 \mathrm{GeV}$ in the muon channel using $35 \mathrm{pb}^{-1}$ of data collected in 2010 (See. Fig. 1).

The dominant uncertainty in the electron channel arises from the imperfect knowledge of the background and varies from $3.9 \%$ to $1.6 \%$ from the lowest to the highest mass bin. In the muon channel the uncertainties are generally smaller by factor of $\sim 1.5$ and dominated by uncertainties due to isolation efficiency corrections.

The comparisons between the measured cross sections and the theoretical predictions are shown in Fig. 2. The FEWZ program at NLO level provides a poor description of the data at low $m$, while the PowHEg predictions differ from FEWZ by $\approx 20 \%$ and agree with the measurement. This improvement 

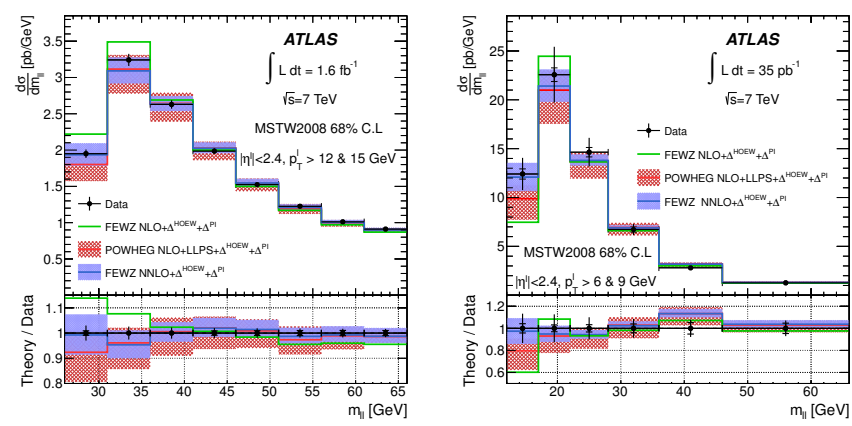

Figure 2. The measured fiducial differential cross section in bins of invariant mass for the nominal analysis (left) and the extended analysis (right) compared to NLO predictions from FEWZ, NLO+LLPS predictions from PowHEg and NNLO predictions from FEWZ. The predictions are calculated using MSTW2008 PDF sets with the appropriate order of perturbative QCD. The uncertainty bands include the PDF and $\alpha_{S}$ variations at $68 \% \mathrm{CL}$, scale variations and the uncertainty in the PI correction. The data (solid points) are displayed at unity with the statistical (inner) and total (outer) measurement uncertainties [2].
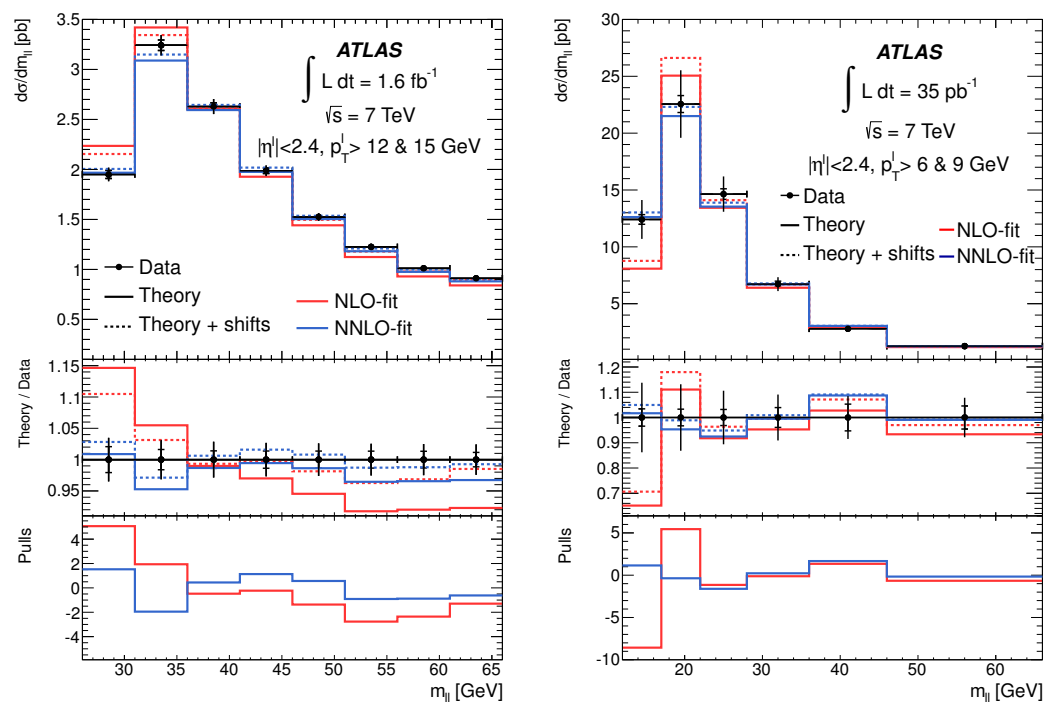

Figure 3. The measured differential cross section in bins of invariant mass, compared to the NLO and NNLO QCD fits. The inner error bars show the total uncorrelated experimental uncertainty, and the outer error bars represent the total experimental uncertainty, excluding the luminosity uncertainties. The lower half of each figure shows the ratio of theory expectations to data in the upper part, and the $\chi^{2}$ pull contribution in the lower part [2].

is due to the cooperation of parton shower effects in the PowHEg production. The pure fixed-order FEWZ predictions at NNLO level also show a good agreement to the measured fiducial cross sections.

The NLO and NNLO QCD fit results are shown in Fig. 3 in the upper panels and compared to the nominal and extended analysis measurements. The results of the fit after applying the adjustments 

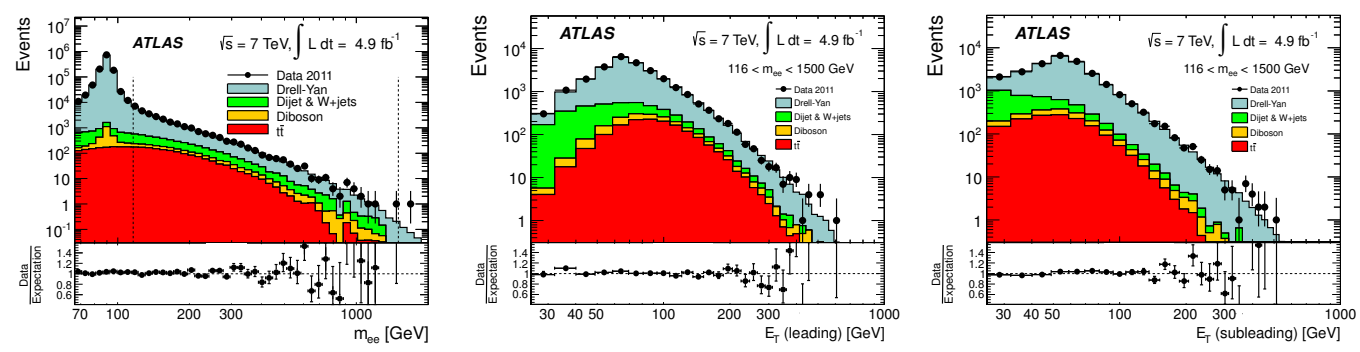

Figure 4. Distribution of $m_{e e}$ (left) and leading and sub-leading electron $E_{T}$ in data compared to the summed signal and background predictions. The Drell-Yan signal is predicted from PутніA simulation and the combined di-jet and $W+$ jets contribution is estimated from data. The dashed vertical lines on the left plot indicate the mass range used for the differential cross-section measurement [3].

of the fitted nuisance parameters for each correlated error source are also shown. The pulls for the NLO fit are large at low invariant masses as expected from the phase space constraints created by the transverse momentum cuts applied to the leptons. The QCD analysis, performed at NNLO, has a good $\chi^{2}$ value of 16.3 for 14 degrees of freedom.

The measurement in a high mass region was performed in electron channel with $4.9 \mathrm{fb}^{-1}$ and covers the mass range of $166-1500 \mathrm{GeV}$. The dominant systematic uncertainties are due to the electron identification for the lower mass bins and the background subtraction in the higher mass bins. The main background components are multi-jet and $W+$ jets events, as it is illustrated in Fig. 4. The statistical uncertainties are larger than the systematic uncertainties for $m_{e e}>400 \mathrm{GeV}$.

The measurement at dressed level was compared to the predictions of the PyTHIA, MC@NLO and SherPa MC generators (See Fig. 5, left). No corrections have been applied to the generatorlevel predictions; instead, the prediction of each generator has been scaled globally to match the total number of events observed in data. The resulting scale factors are 1.23 for PYTHIA, 1.08 for MC@ NLO and 1.39 for Sherpa. The overall shape of the $m_{e e}$ distribution from all three generators is consistent with the data.

Fig. 5 shows the differential cross-section at the Born level compared to calculations in the FEWZ 3.1 framework using various recent NNLO PDFs. The deviations between predictions based on different PDFs are within the total uncertainty band assigned to the MSTW2008 prediction. The predictions show a good compatibility with the data.

\section{Forward-backward asymmetry}

The analysis of $A_{F B}$ is based on $\sqrt{s}=7 \mathrm{TeV}$ ATLAS data collected in 2011, corresponding to an integrated luminosity of $4.8 \mathrm{fb}^{-1}$ for the electron channels and $4.7 \mathrm{fb}^{-1}$ for the muon channel [4].

The electron channel analysis was subdivided to two sub-channels: central-central (CC), where both electron are reconstructed in the central part of the calorimeter and central-forward (CF), where one electron reconstructed in the central calorimeter and the other in the forward. This is due to the electron trigger setup of the ATLAS detector. The unfolded to Born-level distributions of $A_{F B}$ are shown in Fig. 6. Good agreement is observed between the measured and predicted spectra. The CF electron channel demonstrates a larger slope in $A_{F B}$, which leads to a higher sensitivity to the weak mixing angle. 

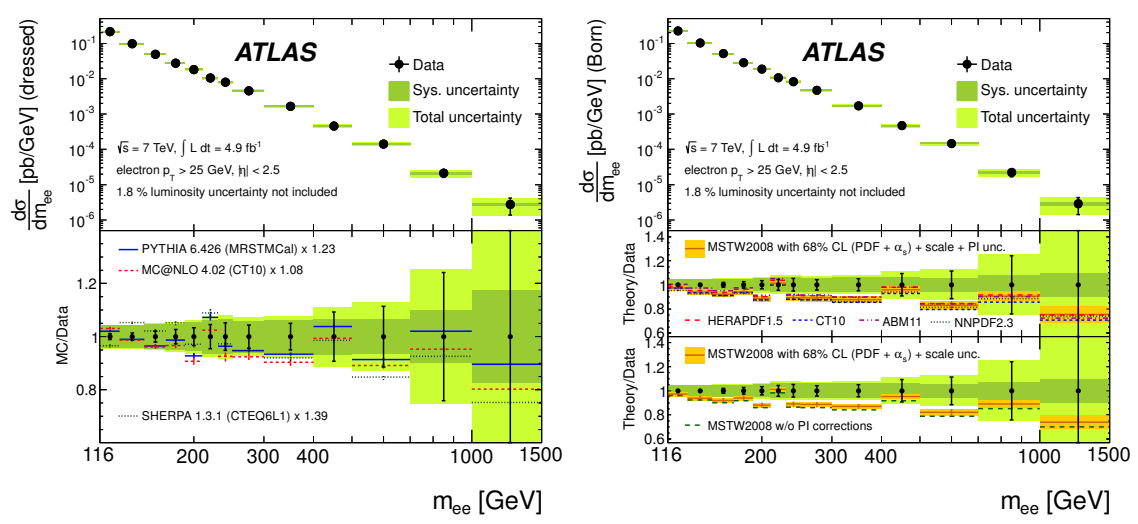

Figure 5. Measured differential cross-section at the dressed level (left) and at the Born level (right) within the fiducial region (electron $p_{T}>25 \mathrm{GeV}$ and $|\eta|<2.5$ ) with statistical, systematic, and combined statistical and systematic (total) uncertainties, excluding the $1.8 \%$ uncertainty on the luminosity. In the left plot, the measurement is compared to the predictions of the PYTHIA, MC@NLO and ShERPA MC generators including their statistical uncertainties. On the right, in the upper ratio plot, the photon-induced (PI) corrections have been added to the predictions obtained from the MSTW2008, HERAPDF1.5, CT10, ABM11 and NNPDF2.3 NNLO PDFs, and for the MSTW2008 prediction the total uncertainty band arising from the PDF, $\alpha_{S}$, renormalisation and factorisation scale, and photon-induced uncertainties is drawn [3].

The final measurements of the leptonic effective weak mixing angle, $\sin ^{2} \theta_{W}^{e f f}$, are based on the raw $A_{F B}$ spectra and were extracted with template-fits. The combined result from the three measurements, assuming lepton universality is

$$
\sin ^{2} \theta_{W \text { combined }}^{e f f}=0.2297 \pm 0.0004 \text { (stat.) } 0.0009 \text { (syst.). }
$$

The systematic uncertainty on the combined result is dominated by PDF uncertainties (0.0007). The results from this analysis, as well as the results from the other collider experiments, are shown in Fig. 7. The combined result of this analysis agrees within $1.8 \sigma$ with the current world average.

\section{$4 Z$ Boson transverse momentum}

The transverse momentum distribution of the $\mathrm{Z}$ boson was measured with $7 \mathrm{TeV}$ ATLAS data directly, using reconstructed spectrum of di-lepton transverse momentum $p_{T, Z}$ [5], but also probed with socalled $\phi^{*}$ variable [6], which has better experimental resolution. The $\phi^{*}$ observable provides a measure of the azimuthal decorrelation between the two objects. The formal definition of $\phi^{*}$ used in the ATLAS analysis is given by

$$
\phi_{\eta}^{*}=\tan \left(\frac{\phi_{\text {acop }}}{2}\right) \sin \theta_{\eta}^{*}, \text { with } \cos \theta_{\eta}^{*}=\tanh \left(\frac{\eta^{-}-\eta^{+}}{2}\right) .
$$

The purpose of this definition of $\theta^{*}$ - as opposed to, for example, that defined in the Collins-Soper frame - is that it does not rely on measurements of lepton momenta, rendering $\theta_{\eta}^{*}$ immune from lepton momenta mis-measurements owing to resolution inefficiencies. Both $p_{T, Z}$ and $\phi^{*}$ measurements are shown in Sec. 4 

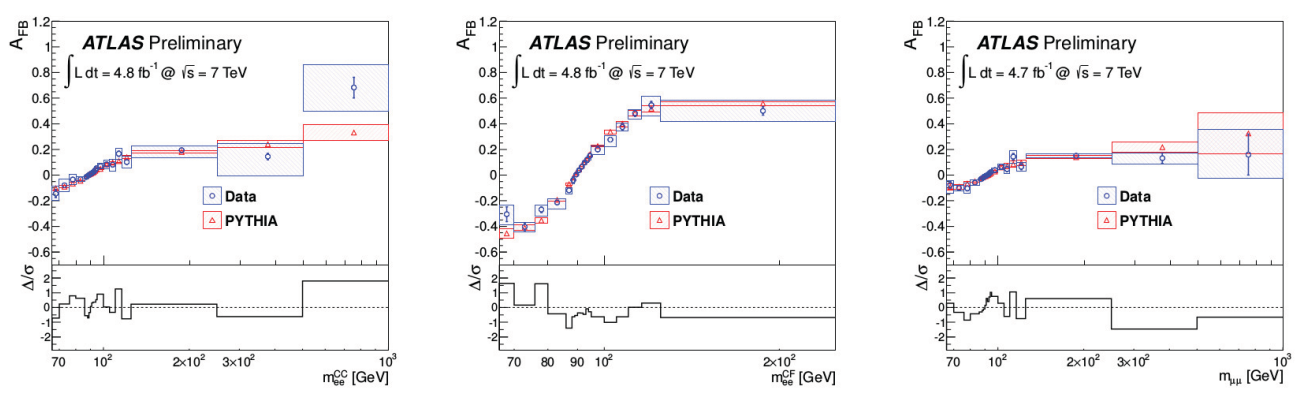

Figure 6. $A_{F B}$ unfolded to Born-level, for the CC electron (left), CF electron (middle) and muon (right) channels. The boxed shaded region on the data shows the total uncertainty with error bars representing the statistical uncertainty, and on the MC represents only the statistical uncertainty. The ratio plots display the distribution of pulls $\Delta / \sigma)$, where $\sigma$ is the quadratic sum of the all of the uncertainties [4].

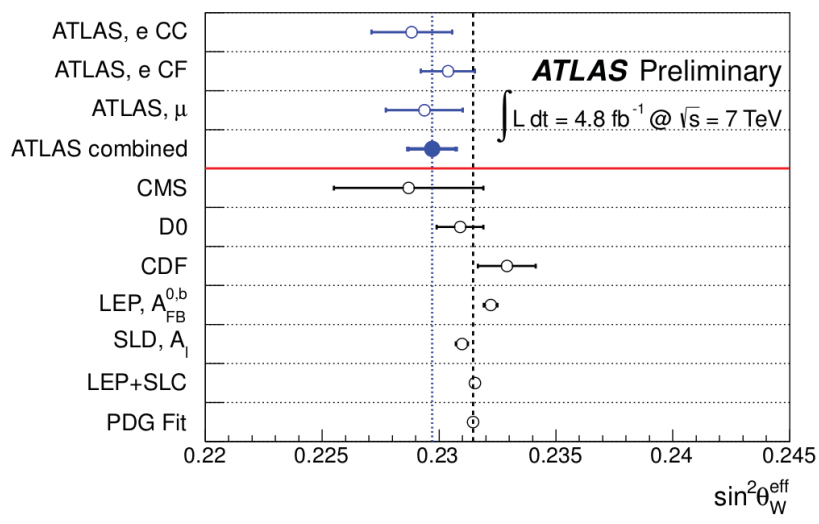

Figure 7. Comparison of the ATLAS results with other published results for $\sin ^{2} \theta_{W}^{e f f}$. A vertical dotted line illustrates the results from the ATLAS combined measurement and a vertical dashed line shows the results from the current PDG global fit [4].
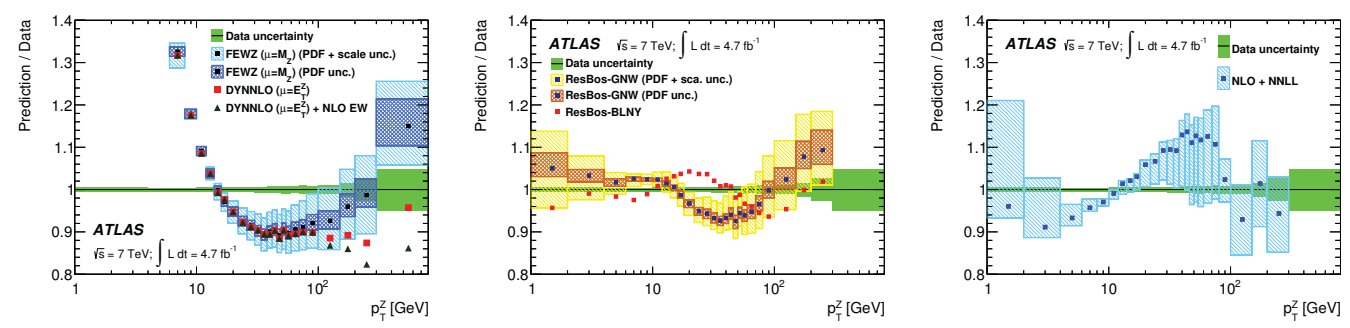

Figure 8. A comparison of the $p_{T, Z}$ distributions predicted by different computations: FEWZ and DynNLo (left), ResBos (middle) and NLO+NNLL (right) with the Born-level combined measurement. The uncertainty on the predictions, estimated from the PDF uncertainties and renormalization and factorization scale variations [5]. 


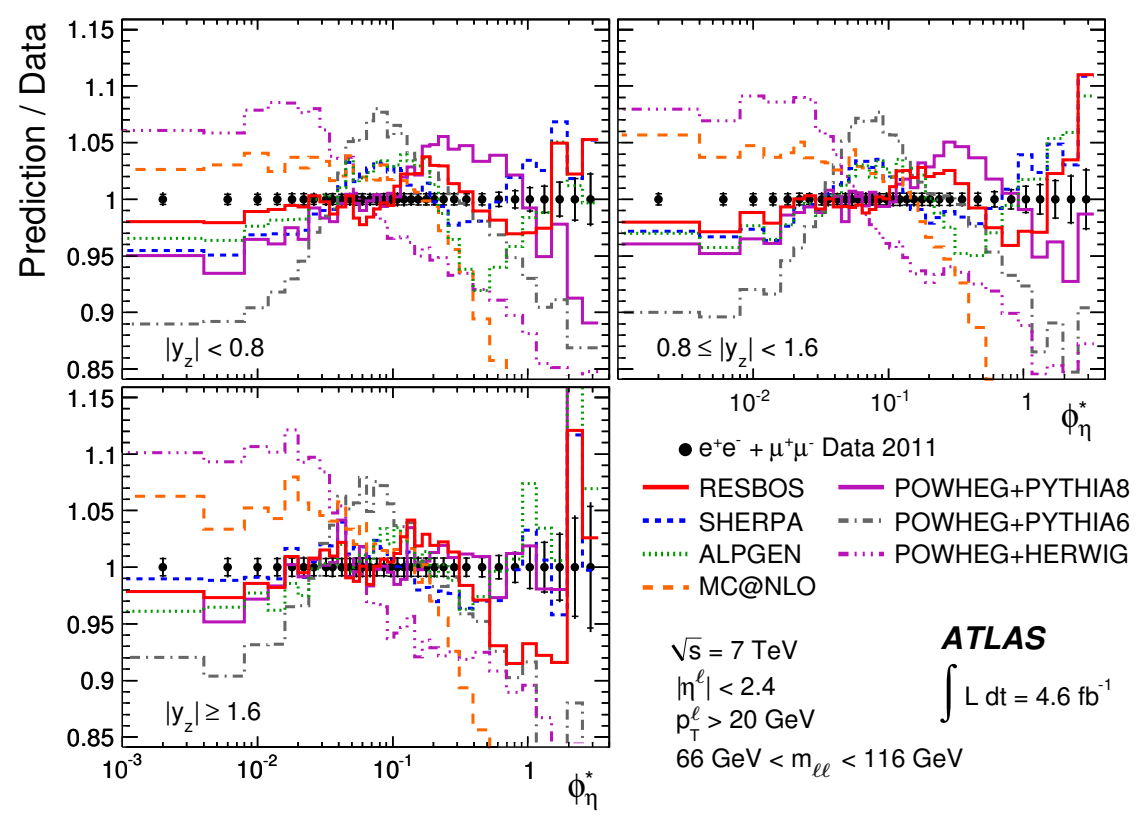

Figure 9. The ratio of the combined normalised differential cross section in bins of $\phi^{*}$ to the ResBos predictions in three ranges of $\left|y_{Z}\right|$. The inner and outer error bars on the data points represent the statistical and total uncertainties, respectively. The uncertainty due to QED FSR is included in the total uncertainties. The measurements are also compared to predictions from different MC event generators [6].

The measurement of $p_{T, Z}$ and $\phi^{*}$ was performed using 2011 ATLAS data in electron and muon decay channels. The analysed events were selected in the peak mass region $(66--116 \mathrm{GeV})$ and the distributions were measured as well inclusively as in three boson rapidity bins $\left(y_{Z} \in[0.0,0.8,1.6,2.4]\right)$. The combined result at born-level, integrated over $y_{Z}$, is shown in Fig. 8 and have been compared to theoretical predictions from FEWZ, DynnLo, ResBos (based on the CT10 PDF-Set) and NLO+NNLL Calculations (based on the CTEQ6m PDF-set). As it can be seen, the ResBos-GNW prediction agrees with the data within 5-7\%, the NLO+NNLL calculation matches the data within 10-12\%.

The ratio of the combined normalised differential cross section vs $\phi^{*}$ to the ResBos prediction is shown for the three $\left|y_{z}\right|$ ranges in Fig. 9. The measurement is also compared to predictions obtained using different MC event generators. The CT10 PDF set is employed in all calculations, except for the AlPGEn prediction, where the CTEQ6L1 PDF set was used. The Sherpa generator describes the data better than ResBos over all $\left|y_{Z}\right|$ bins for $\phi^{*}>0.1$. However, for $\phi^{*}<0.1$ the deviations of Sherpa or Alpgen from the data are $~ 5 \%$ and hence larger than those of ResBos. The Powheg generator interfaced to Pyтнia8 is also able to describe the data to within $5 \%$ over the whole $\phi^{*}$ range.

In a second step, the $p_{T, Z}$ and $\phi^{*}$ measurements were used to tune the Pythia 8 and PowHEG+PYTHIA8 generators. Both measurements can be interpreted consistently in terms of the $Z / \gamma^{*}$ bosons transverse momentum distribution and provide similar sensitivity to parton shower model parameters. In order to avoid correlations between these measurements, the final tune optimally combines the most precise independent single measurements, namely the muon channel $p_{T, Z}$ measurement, and the electron channel $\phi^{*}$ measurement. 

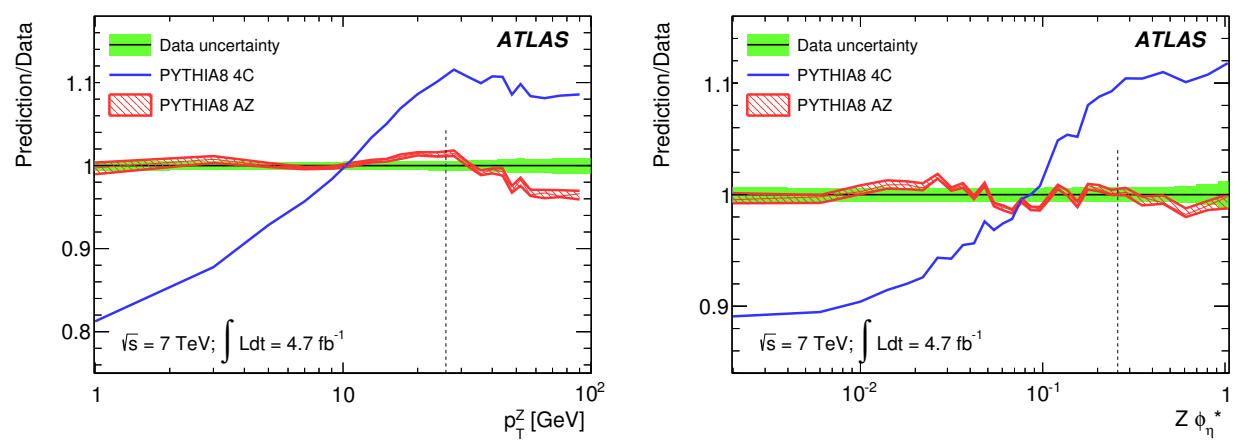

Figure 10. Comparison of tuned predictions to the $p_{T, Z}$ and $\phi^{*}$ differential cross sections, for dressed kinematics and in the full rapidity range. Comparison of the Рүтніa 8 generator with the $4 \mathrm{C}$ and $\mathrm{AZ}$ tunes to the muonchannel $p_{T, Z}$ data (left) and electron-channel $\phi^{*}$ data (right). The vertical dashed lines show the upper limit of the tuning range. [5].

The final comparison of the PYтHIA8 generator with the corresponding tunes to the muon-channel $p_{T, Z}$ and electron-channel $\phi^{*}$ are presented in Fig. 10. The tuned predictions agree with the measurement to better than $2 \%$ in the range that has been used for the tuning (below $p_{T, Z}=50 \mathrm{GeV}$ ). At higher transverse momentum, discrepancies of around $15 \%$ for Pythia8 remain, indicating the limited accuracy of the NLO signal matrix element and suggesting the need for contributions from higher parton multiplicity.

\section{Conclusion}

The first run of the LHC allowed for several precision measurements of the production cross-section of the vector bosons in proton-proton collisions in a new energy regime. The properties of $Z / \gamma^{*}$ production at ATLAS were measured with high accuracy using high statistics data with millions of $Z$ events. The inclusive and differential $Z \rightarrow l l$ cross-section measurements are sensitive to PDFs and provide new constraints for future PDF-sets. The measurement of $Z$-boson transverse momentum has been used to tune parton shower models and tests $\mathrm{pQCD}$ calculations. Last but not least, also a first measurement of a fundamental electroweak parameter, namely the leptonic effective weak mixing angle, was performed at the LHC. Its determination is based on the measurement of the forwardbackward asymmetry $A_{F B}$ and shows good agreement with the current PDG global fit value. In the near future, significant improvements in all areas of QCD and electroweak precision measurements are expected during the second run of the LHC in the years 2015 and 2016.

\section{References}

[1] Schott, M. and Dunford, M., Eur.Phys.J.C74, arXiv:hep-ex 1405.1160

[2] ATLAS Collaboration, JHEP 1406 (2014) 112

[3] ATLAS Collaboration, Phys. Lett. B 719 (2013) 242-260

[4] ATLAS Collaboration, ATL-CONF-2013-043, https://cds.cern.ch/record/1544035

[5] ATLAS Collaboration, arXiv:1406.3660

[6] ATLAS Collaboration, Phys. Lett. B 720 (2013) 32-51 